\title{
Isthmic spondylolisthesis treated with circumferential arthrodesis (ALIF and posterior fixation): correction, fusion and indirect decompression
}

\author{
M. Khalifé, C. Dauzac, T. Lenoir, B. Magrino
}

From the Clinique Médico-Chirurgicale Ambroise Paré, Neuilly-sur-Seine, France

There is no consensus regarding the choice of the surgical technique for isthmic spondylolisthesis treatment, although they all aim to a common goal, achieving fusion at the index level while restoring an appropriate lordosis and remove potential radicular compression. Analyze outcome of circumferential arthrodesis (CA) with ALIF (Anterior Lumbar Interbody Fusion) and pedicle screw fixation for the treatment of all-grade isthmic spondylolisthesis, with indirect neurological decompression. Retrospective study of isthmic spondylolisthesis treated with CA, with one-year follow-up. Clinical scores were collected at one year: VAS-L, VAS-R and ODI. Pelvic parameters, L4-S1 lordosis and at index and adjacent levels, and lumbo-sacral angle (LSA) were measured pre- and post-operatively and at last follow-up. Foraminal surface and diameters were measured preoperatively and at follow-up on CT-Scan. Level of evidence: IV. 87 patients were included. Mean VAS-L was 2.3, mean VAS-R was 1 , and mean ODI was $13.8 \% .10 \%$ of the patients presented a high-grade spondylolisthesis and $\mathbf{5 0 \%}$ a grade II. Mean lordosis at index level shifted from $6^{\circ}$ to $18^{\circ}, \mathrm{L4}-\mathrm{S} 1$ lordosis increased from 37 to $45^{\circ}$ and LSA shifted from 116 to $125^{\circ}(\mathrm{p}<0.001)$. The foraminal surface increased from $50 \mathrm{~mm}^{2}$ to $70 \mathrm{~mm}^{2}$ at last follow-up mostly through the supero-inferior diameter, shifting from $7.4 \mathrm{~mm}$ to $9.5 \mathrm{~mm}(\mathrm{p}<\mathbf{0 . 0 0 1})$. In $\mathrm{LSA}<90^{\circ}$ group, mean correction was $20^{\circ}$ at index level, $13^{\circ}$ at L4-S1 and $21^{\circ}$ for LSA versus $11^{\circ}, 8^{\circ}$ and $8^{\circ}$ respectively in $\mathrm{LSA}>90^{\circ}$ group $(p<0.001)$. Fusion rate was estimated at $96.5 \%$. One infection, 5 sympathetic dysfunctions, one retrograde ejaculation, one iliac vein injury, one incisional

No benefits or funds were received in support of this study. None of the authors have a conflict of interest. hernia, one lateral femoral cutaneous nerve injury and two adjacent syndromes have been noted. CA is an efficient technique for the treatment of isthmic spondylolisthesis of all grades, with an acceptable rate of complications. It allows a restoration of the regional lordosis as well as a foraminal widening, avoiding additional decompression.

Keywords : Isthmic spondylolisthesis ; circumferential fusion ; indirect decompression.

\section{INTRODUCTION}

Isthmic spondylolisthesis mainly affects young adults, with a prevalence estimated around 6\% (1). The theory of repeated micro-traumatisms seems to be the most likely, causing a stress fracture that does not heal and leads to an isthmic nonunion. Risk

\footnotetext{
M. Khalifé ${ }^{1,2}, \mathrm{MD}, \mathrm{MSc}$,

C. Dauzac ${ }^{1}, \mathrm{MD}$,

T. Lenoir ${ }^{1,2}, \mathrm{MD}$,

B. Magrino ${ }^{1}, \mathrm{MD}$

${ }^{1}$ Clinique Médico-Chirurgicale Ambroise Paré, Neuilly-surSeine, France.

${ }^{2}$ Hôpital Européen Georges Pompidou. Paris, France.

Correspondence : Correspondence: Marc Khalifé, MD, MSc. ORCID: 0000-0001-9022-3336, Phone : +33685574242.

Email : marc.khalife@aphp.fr

- 2021, Acta Orthopædica Belgica.
} 
factors such as a high pelvic incidence or the practice of certain sports as a child have been identified $(2,3)$.

After an unsuccessful well-conducted medical management, a surgical solution can be offered. There are numerous ways to achieve the arthrodesis such as stand-alone ALIF (Anterior Lumbar Interbody Fusion), isolated postero-lateral fusion, TLIF (Transforaminal Lumbar Interbody Fusion), PLIF (Posterior Lumbar Interbody Fusion), or a combined approach, through a traditional or minimally invasive approach. This condition is not rare, however there is no consensus regarding the choice of the surgical technique, although they all aim to three common goals: achieving fusion at the index level while restoring an appropriate lordosis and remove potential radicular compression (4). According to several authors, circumferential arthrodesis is the best option to reach these goals, especially in terms of fusion (5-7).

The objective of this study was to analyze the results of circumferential arthrodesis starting with an ALIF, completed with pedicle screw fixation for the treatment of all-grade isthmic spondylolisthesis, with no direct neurological decompression associated, in terms of radiographic correction, foramen widening and clinical outcome.

\section{MATERIALS \& METHODS}

\section{Population}

Retrospectively, the records of consecutive patients operated by three surgeons of one single center between 2015 and 2017 have been analyzed. Patients have been included if they had been operated on for an isthmic spondylolisthesis through a combined approach, at the L4-L5 or L5-S1 level, with a one-year minimum post-operative follow-up. All patients presented preoperative leg pain, uni- or bilateral, in the L4 or L5 territory. Patients have not been included if they had previously been operated at the level of the spondylolisthesis, if they were aged under 18 years or had language comprehension issues. Prior consents of included patients have been collected and local ethics committee gave its approbation for the study.

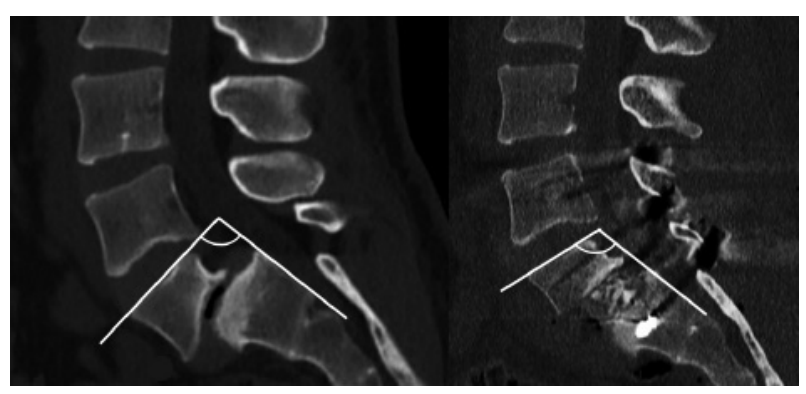

Fig. 1. - CT-scan sagittal view of a grade III spondylolisthesis, before and after surgery. Sacral dome and anterior lower corner of L5 osteotomies have been performed. Lumbo-sacral angle increased from $85^{\circ}$ to $109^{\circ}$.

\section{Surgical technique}

All included patients have been operated on following the same surgical technique in two-stage surgery, performed consecutively the same day:

- First stage: ALIF

In "French" position - supine position, lower limbs spread -, a retro-peritoneal approach was performed through Pfannenstiel incision for L5-S1 level, or median sub-umbilical for L4-L5 level. After discectomy, a polyetheretherketone (PEEK) cage was put in place between the two vertebral bodies, stabilized using either anchors or integrated plate with screws. This cage was filled either with cancellous bone graft harvested from the iliac crest,

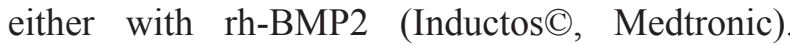
The aim of this first stage was to restore disc height in order to perform indirect decompression, with a wide graft surface to achieve bone fusion, and kyphosis correction whereas degree of slip was not taken into consideration. For high grade spondylolistheses, osteotomy of the lower anterior corner of L5 vertebra was often required to access interbody space, sometimes associated with sacral dome osteotomy to flatten sacral promontory (Fig. $1)$.

\section{- Second stage : Posterior fixation}

The patient was installed in prone position. Through a median posterior approach, a posterior fixation was performed using pedicle screws. L5 

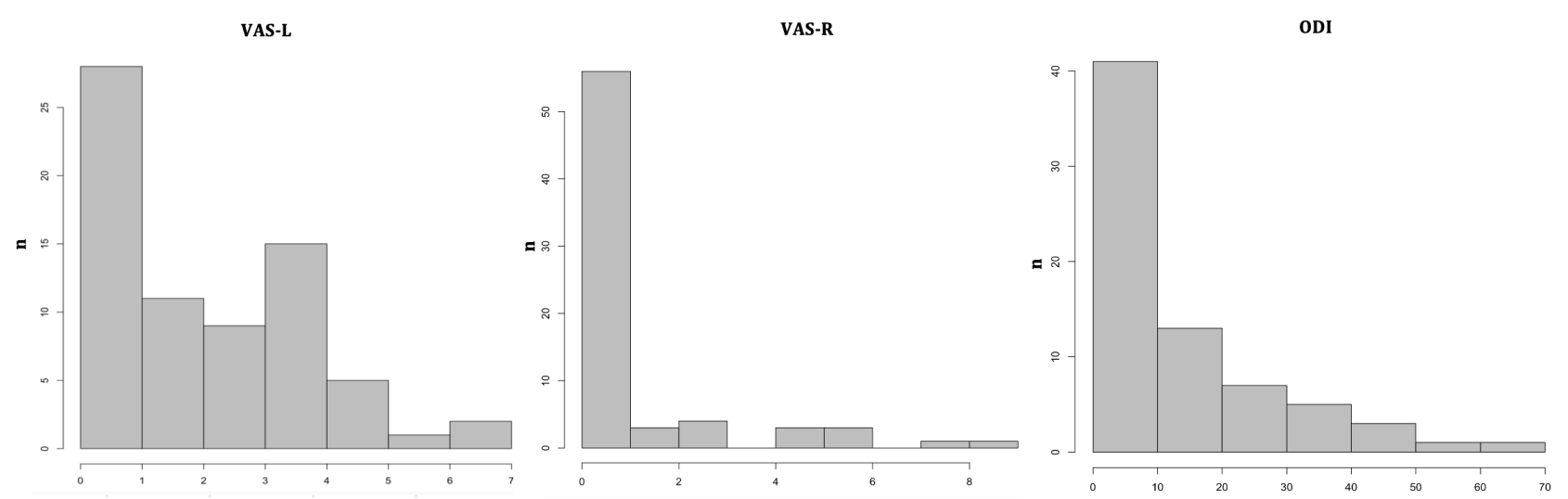

Fig. 2. - Clinical scores at last follow-up.

spinous process was used as autograft (or L4 in case of L4-L5 fusion). No associated decompression was performed, neither central nor foraminal, the Gill fragment was kept in place to avoid decompression complications.

No brace was worn post-operatively. A two-level arthrodesis has been performed in case of a very kyphotic deformity.

\section{Analysis parameters}

Usual demographic data have been collected, such as age, gender, weight, height and body mass index (BMI). On the clinical standpoint, presence of leg pain and a sensitive or motor deficit in the lower limbs have also been noted. At last follow-up - at one-year post-operative - the lumbar visual analog scale (VAS-L) and radicular (VAS-R), as well as the Oswestry Disability Index (ODI) and the patient's satisfaction after surgery have been collected.

The surgical data of interest were the dimensions of the implanted cage, in terms of height and lordosis as well as the use of iliac crest graft or rh-BMP2, and if a double-level arthrodesis was performed. The length of hospital stay and the occurrence of intra- or post-operative complications have been studied as well.

Pre-operative, post-operative and at last followup X-rays have been analyzed using GXD5 PACS software. Pre-operatively, the level of the spondylolisthesis, the grade according to Meyer- ding's classification and the presence of transitional lumbo-sacral vertebrae have been noted.

At the three time points of analysis, the lordosis at the level of the spondylolisthesis (measured between the inferior plate of the lower vertebra and the superior plate of the upper vertebra), the lordosis at the adjacent level, the L4-S1 lordosis (measured between the superior plate of L4 and the sacral plate) and the lumbo-sacral angle, as described by Dubousset (8), (measured between the superior plate of the slipped vertebra and the tangent to the posterior wall of S1 (Fig. 1)) have been measured. Pre-operatively and at last followup, the pelvic parameters have been measured, namely pelvic incidence (PI), pelvic tilt (PT) and sacral slope (SS); and on the CT-scan images, when available, the antero-posterior and cephalad-caudad foramen diameters as well as the foraminal surface have been measured. Lastly, the presence of fusion was assessed on the CT-scan at last follow-up when available by one surgeon and one independent observer. The fusion was defined by the presence of at least one osseous bridge between the two vertebral bodies at the level of the arthrodesis, and the absence of instability signs such as screws loosening, hardware displacement or breakage, on $\mathrm{X}$-Rays or CT-scan.

First, a descriptive analysis of the different studied parameters has been performed. Comparisons of these variables between the different measurement time-points have then been carried out, using paired 
Table 1. - Demographic and clinical data

\begin{tabular}{|l|c|c|c|c|c|c|}
\hline Demography & Mean & Std dev. & Mini. & Maxi. & Median & $\boldsymbol{n}$ \\
\hline Age & $\mathbf{4 8}$ & 11,3 & 22 & 77 & 47 & 87 \\
\hline BMI & $\mathbf{2 5 . 6}$ & 4 & 18 & 39.7 & 25.2 & 86 \\
\hline Clinical & & & & & & \\
\hline VAS-L & $\mathbf{2 . 3}$ & 2 & 0 & 7 & 2 & 72 \\
\hline VAS-R & $\mathbf{1}$ & 2.1 & 0 & 9 & 0 & 72 \\
\hline ODI & $\mathbf{1 3 . 8}$ & 14.6 & 0 & 66 & 8 & 72 \\
\hline
\end{tabular}

Table 2. - Radiological data expressed by means \pm standard deviations

\begin{tabular}{|c|c|c|c|c|c|}
\hline & Pre-operative & Post-operative & Follow-up & $p$ & $n$ \\
\hline Index lordosis $\left({ }^{\circ}\right)$ & $6 \pm 9.7$ & $18 \pm 5.2$ & $18 \pm 6.2$ & $<0.0001 *$ & 66 \\
\hline Adjacent lordosis $\left(^{\circ}\right)$ & $13 \pm 6.4$ & $10 \pm 4.7$ & $11 \pm 4.8$ & 0.12 & 66 \\
\hline L4-S1 lordosis $\left(^{\circ}\right)$ & $37 \pm 10.1$ & $45 \pm 8.2$ & $45 \pm 10.9$ & $<0.0001 *$ & 66 \\
\hline Lumbo-sacral angle $\left(^{\circ}\right)$ & $116 \pm 17$ & $127 \pm 12.4$ & $125 \pm 12.6$ & $<0.0001 *$ & 66 \\
\hline Pelvic tilt $\left(^{\circ}\right)$ & $20 \pm 8.7$ & - & $17 \pm 7.2$ & $0.004 *$ & 51 \\
\hline Sacral slope $\left(^{\circ}\right)$ & $48 \pm 9.7$ & - & $51 \pm 8.8$ & $0.04 *$ & 51 \\
\hline Left foraminal surface (mm2) & $53 \pm 29.8$ & - & $69 \pm 23.5$ & $<0.0001^{*}$ & 35 \\
\hline Right foraminal surface (mm2) & $50 \pm 23.1$ & - & $70 \pm 24.3$ & $<0.0001^{*}$ & 35 \\
\hline $\mathbf{L}$ foramen AP diameter (mm) & $8.4 \pm 2$ & - & $8.9 \pm 2$ & $0.002 *$ & 35 \\
\hline L foramen CC diameter (mm) & $7.4 \pm 3.7$ & - & $9.5 \pm 3.1$ & $<0.0001^{*}$ & 35 \\
\hline $\mathbf{R}$ foramen AP diameter (mm) & $8.7 \pm 2$ & - & $9 \pm 1.4$ & 0.07 & 35 \\
\hline $\mathbf{R}$ foramen $\mathbf{C C}$ diameter (mm) & $6.9 \pm 3.4$ & - & $9.3 \pm 3.2$ & $<0.0001 *$ & 35 \\
\hline
\end{tabular}

L: Left. R: Right. AP: Antero-posterior. CC: Cephalad-caudad.

Student $t$ tests. Lastly, a comparative analysis of the correction surgically achieved has been performed according to the pre-operative lumbo-sacral angle, depending on whether it was higher or lower than $90^{\circ}$.

P-values lower than 0.05 defined statistical significance. No p-value adjustments were made as multiple comparisons were generating hypothesis. Statistical analyses have been carried out using RStudio software (RStudio Inc., version 1.1.423).

\section{RESULTS}

Eighty-seven patients have been included in the study. Mean age was 48 years [22-77], with a slight predominance of female patients in the cohort representing 56\%. Mean BMI was $25.5 \mathrm{Kg} . \mathrm{m}^{-2}$ [1839.7], with half of the cohort overweight (Fig. 2). Almost $19.5 \%$ of the patients presented a lower limb neurological deficit pre-operatively.

Regarding clinical scores, available for 72 patients, mean VAS-L was 2.3 [0-7], mean VAS-R was 1 [0-9], and mean ODI was 13.8\% [0-66] (Fig. 2 - Table 1). Patients expressed satisfaction regarding their choice of surgical management, except one patient $(1.4 \%)$ who estimated he was not relieved enough after surgery.

For $94 \%$ of the patients, the cage has been filled with cancellous bone graft harvested from the 


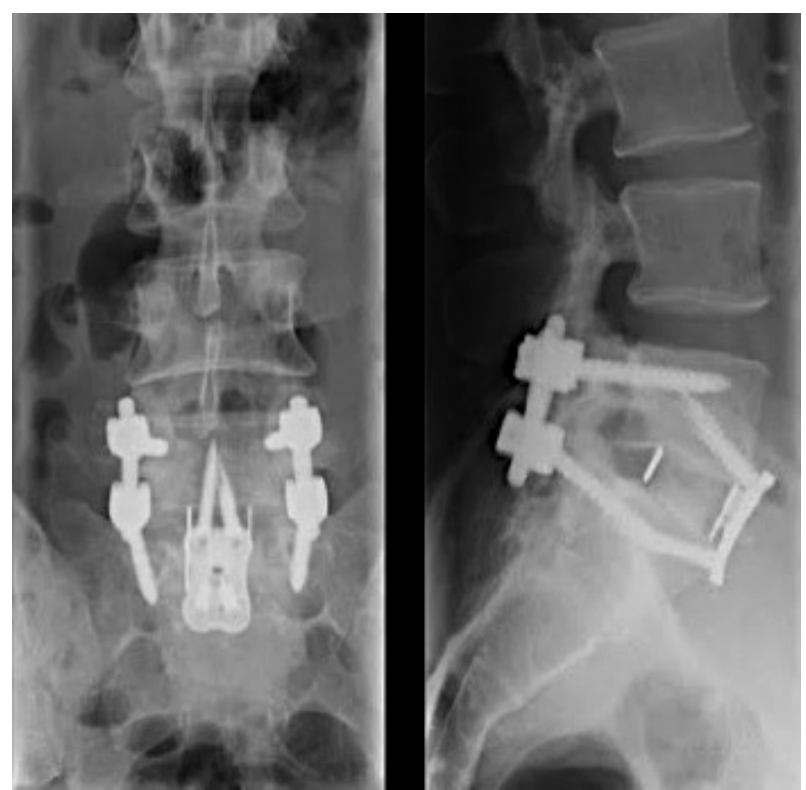

Fig. 3. - High-grade spondylolisthesis, treated with circumferential arthrodesis at L5-S1 level, and posterior fixation at L4-L5.

iliac crest, rh-BMP2 has been used in only $6 \%$ of the patients. The most used cages were of 14- or 12-millimeters height [8-16], respectively 40 and $36 \%$, and of $14-$ and 10-degrees lordosis [6-16], respectively 47 and $35 \%$. One arthrodesis of the two last mobile levels has been performed in 6 patients (7\%). In average, patients were discharged at fourth day post-operative [3-6].

Regarding complications, one surgical site infection of the posterior approach has been noted, managed by further intervention of wound debridement. Related to the anterior approach, five patients declared having sympathetic dysfunction in the lower limbs (5.7\%). Further, one retrograde ejaculation, one iliac vein injury, one incisional

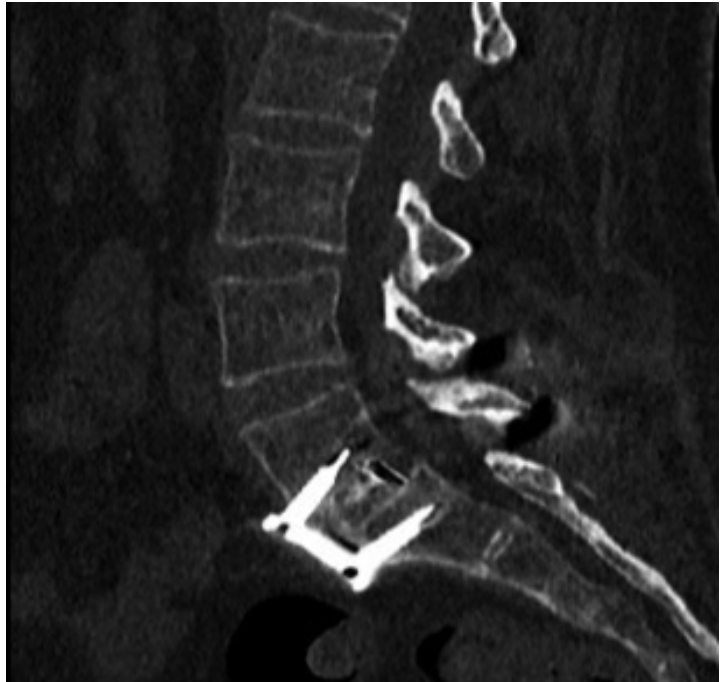

Fig. 4. - L5-S1 fusion on sagittal CT-Scan image.

hernia and one retro-peritoneal hematoma that required radio-embolization, have been identified (1.1\% per complication). Two adjacent syndromes have also been identified $(2.3 \%)$, one treated with rhizolysis, the second required revision surgery with extension of the arthrodesis to L4-L5 at one year post-operative.

\section{Radiological data}

Sixty-six patients had complete radiographic data and 35 had CT-Scan at one year follow-up. Ten patients presented a lumbo-sacral transitional anomaly $(11.5 \%)$. According to Meyerding's classification, 35 patients presented a grade 1 spondylolisthesis (40\%), 43 patients were graded $2(50 \%)$, 8 patients presented a grade 3 spondylolisthesis $(9 \%)$ and one patient a spondyloptosis (Fig. 3). The

Table 3. - Correction comparison between LSA $>90^{\circ}$ and $\operatorname{LSA}<90^{\circ}$

\begin{tabular}{|l|c|c|c|}
\hline & LSA $<\mathbf{9 0}^{\circ}$ & LSA $>90^{\circ}$ & $\boldsymbol{p}$ \\
\hline $\boldsymbol{n}$ & 7 & 59 & - \\
\hline Index level correction $\left(^{\circ}\right)$ & $19.6 \pm 11.9$ & $10.9 \pm 6.53$ & $<0.001^{*}$ \\
\hline Adjacent level correction $\left(^{\circ}\right)$ & $-1.3 \pm 11.12$ & $-3.5 \pm 7.39$ & $<0.001^{*}$ \\
\hline L4-S1 correction $\left(^{\circ}\right)$ & $13.1 \pm 9.42$ & $7.8 \pm 8.67$ & $<0.001^{*}$ \\
\hline LSA correction $\left(^{\circ}\right)$ & $21.1 \pm 12.9$ & $7.6 \pm 7.49$ & $<0.001^{*}$ \\
\hline
\end{tabular}


spondylolisthesis was located at the L5-S1 level in 68 patients (78\%), and L4-L5 in 19 patients (22\%).

Mean lordosis at the index level was $6^{\circ}$ preoperatively. It significantly increased postoperatively and at last follow-up at $18^{\circ}(\mathrm{p}<0.0001)$. Similarly, L4-S1 lordosis increased significantly from 37 to $45^{\circ}(\mathrm{p}<0.0001)$, as well as lumbo-sacral angle, which shifted from 116 to $125^{\circ}$ at last followup $(p<0.0001)$. Conversely, mean adjacent level lordosis decreased from $13^{\circ}$ preoperatively to $11^{\circ}$ at follow-up $(\mathrm{p}=0.12)$. Pelvic tilt also decreased from $20^{\circ}$ preoperatively to $17^{\circ}$ at last follow-up $(\mathrm{p}=0.004)$, and inversely, sacral slope shifted from 48 to $51^{\circ}$ at follow-up $(\mathrm{p}=0.04)$.

Foraminal analysis highlighted an increase of the foraminal surface measured at 50 and $53 \mathrm{~mm}^{2}$ respectively on the right and left side preoperatively, versus 70 and $69 \mathrm{~mm}^{2}$ at last followup $(p<0.0001)$. Foraminal surface mostly increased on the supero-inferior diameter, gaining $2.1 \mathrm{~mm}$ on the left side, shifting from 7.4 to $9.5 \mathrm{~mm}(\mathrm{p}<0.0001)$; and $2.4 \mathrm{~mm}$ on the right side, shifting from 6.9 to $9.3 \mathrm{~mm}(\mathrm{p}<0.0001)$. The antero-posterior diameter was increased by 0.3 and $0.5 \mathrm{~mm}$ at last follow-up respectively on the right and on the left side ( $p=0.07$ and $\mathrm{p}=0.002$ respectively). All the radiological findings are reported in Table 2.

Regarding the fusion rate, it has been estimated at $96.5 \%$ (Fig. 4). Two cases of non-union have been identified (3.5\%). One of them showed no osseous bridges between the two vertebral bodies on the CTscan images, the other one exhibited a pedicle screw fracture, visible on plain X-Rays. However, clinical outcome remained favorable with ODI scores at respectively 10 and 6 .

\section{Comparative analysis: Lumbo-sacral angle (LSA) inferior or superior to $90^{\circ}$}

In the studied cohort, 7 patients had an LSA inferior to $90^{\circ}$ whereas 59 had an LSA superior to $90^{\circ}$ (Table 3). Surgical correction was significantly greater in the most kyphotic group. Indeed, at the index level, mean correction was $20^{\circ}$ in $\mathrm{LSA}<90^{\circ}$ group versus $11^{\circ}$ in $\mathrm{LSA}>90^{\circ}(\mathrm{p}<0.001)$. Similarly, L4-S1 lordosis was increased by $13^{\circ}$ in the LSA $<90^{\circ}$ group versus $8^{\circ}(\mathrm{p}<0.001)$ as well as the LSA which was increased by $21^{\circ}$ versus $8^{\circ}$ in the LSA $>90^{\circ}$ group $(\mathrm{p}<0.001)$. At the adjacent level, lordosis decreased by $3^{\circ}$ in LSA $>90^{\circ}$ group versus $1^{\circ}$ in the LSA $<90^{\circ}(\mathrm{p}<0.001)$.

\section{DISCUSSION}

Although there is no consensus regarding which surgical technique to choose for the treatment of isthmic spondylolisthesis, several authors demonstrated that the combination of arthrodesis techniques was more efficient than an isolated approach for the correction and the achievement of fusion. Among them Boissière et al. who demonstrated good clinical and radiological outcomes after circumferential fusion in patients with isthmic spondylolisthesis or degenerative disc diseases (9). Zhang et al. in 2015, and Luo et al. in 2017 demonstrated that adding PLIF cages to postero-lateral fusion allowed a greater reduction, as well as a greater disc height and segmental lordosis $(10,11)$. Wang et al. found a higher fusion rate in combined approaches comparing to TLIF and standalone ALIF (4). Tye et al. exhibited better clinical and radiological outcomes for double approaches comparing to TLIF (12). Farah et al. showed good outcomes with ALIF and percutaneous pedicle fixation (13).

This study showed that the circumferential arthrodesis was an efficient solution in the treatment of isthmic spondylolisthesis of any grade, in order to correct local kyphosis, achieve fusion and perform an indirect decompression of nerve roots.

Regarding clinical outcome, the scores collected were satisfactory with a mean VAS-L and VAS-R at last follow-up respectively at 2.3 and 1 . These results are comparable with the series of Farah et al. which included 43 patients treated with minimallyinvasive surgery, that found respectively 2 and 1 for VAS-L and VAS-R (13). Mean ODI at followup in this study was $13.8 \%$ whereas Koné et al., in a similar cohort of 27 patients, found a mean ODI at $28.7 \%$ (14). A study by Tamburrelli et al. analyzed clinical scores of patients with isthmic spondylolisthesis treated with minimally invasive surgery found a mean ODI and VAS-L respectively at $24.1 \%$ and $2.2(15)$. 
Mean hospital stay was 4 days, which is comparable to series of patients treated with minimally invasive surgery ${ }^{13}$.

In this study, lordosis at the level of the spondylolisthesis as well as L4-S1 lordosis and lumbosacral angle were all significantly increased, by respectively $+200 \%,+22 \%$ and $+6 \%$. Conversely, adjacent level lordosis decreased at last follow-up by $15 \%$, non-significantly. This can be explained by the fact that the adjacent disc has been compensating for the lordosis deficit related to the spondylolisthesis, that has been surgically corrected. The correction of lordosis deficit has also been demonstrated by the change of pelvic tilt, which was significantly lower at follow-up compared to pre-operatively, by 3 degrees $(15 \%)$. Correction was significantly greater in most kyphotic patients, with a lumbo-sacral angle inferior to $90^{\circ}$ than those with LSA $>90^{\circ}(\mathrm{p}<0.001)$.

In a 2018 meta-analysis comparing different surgical techniques, Noorian et al. demonstrated that the reduction of vertebral slip did not improve clinical outcome, which supports the choice that has been made here not to analyze vertebral slip (16).

This study demonstrates the important indirect foraminal decompression achieved through the increase of disc height only, as no direct decompression procedure has been performed. Thus, foraminal surface was significantly increased postoperatively, between 16 and $20 \mathrm{~mm}^{2}(30-40 \%$ increase). Expectedly, it is the cephalad-caudad diameter that increased the most, about $2.2 \mathrm{~mm}$ (about 30\% increase). Antero-posterior foramen diameter also increased, to a lesser extent, about $0.4 \mathrm{~mm}$.

This increase of the foraminal space measured on CT-scan images, associated with the good clinical outcome regarding VAS-R seem to indicate that no associated decompression procedure is necessary, regardless of spondylolisthesis grade. This interesting point of the study is supported by Jacobs et al. who drew the same conclusion about low-grade spondylolisthesis (17), and most recently Farah et al. (13). Carragee, in a prospective study, highlighted an association between decompression procedure and increase of non-union risk, and unsatisfactory outcome (18).
This study has some limitations, inherent to its retrospective character. Indeed, clinical scores were not available pre-operatively but only at last followup, for 72 patients. Furthermore, all the radiological data was not available, which allowed to perform foraminal study for 35 patients of the cohort and the radiographical study for 66 patients.

\section{CONCLUSION}

Circumferential arthrodesis through a combined approached starting with an ALIF followed by a pedicle screw fixation is an efficient technique for the treatment of isthmic spondylolisthesis of all grades. It allows a restoration of the regional lordosis as well as a foraminal widening, and high fusion rate, avoiding additional decompression procedure; with an acceptable rate of complications.

Acknowledgment: We thank Cécile Naudin, phd (RICAP) for her help in setting up the study.

\section{REFERENCES}

1. Beutler WJ, Fredrickson BE, Murtland A, et al. The natural history of spondylolysis and spondylolisthesis: 45year follow-up evaluation. Spine (Phila Pa 1976) 2003; 28: 1027-35; discussion 1035.

2. Wiltse LL, Widell EH, Jackson DW. Fatigue fracture: the basic lesion is inthmic spondylolisthesis. J Bone Joint Surg Am 1975; 57: 17-22.

3. Labelle H, Roussouly $\mathrm{P}$, Berthonnaud E, eet al. Spondylolisthesis, pelvic incidence, and spinopelvic balance: a correlation study. Spine (Phila Pa 1976) 2004; 29: 2049-54.

4. Wang S-J, Han Y-C, Liu X-M, et al. Fusion techniques for adult isthmic spondylolisthesis: a systematic review. Arch Orthop Trauma Surg 2014; 134: 777-84.

5. Heary RF, Bono CM. Circumferential fusion for spondylolisthesis in the lumbar spine. Neurosurg Focus 2002; 13: E3.

6. Spruit M, Pavlov PW, Leitao J, et al. Posterior reduction and anterior lumbar interbody fusion in symptomatic low-grade adult isthmic spondylolisthesis: short-term radiological and functional outcome. Eur Spine J 2002; 11: 428-33.

7. Agabegi SS, Fischgrund JS. Contemporary management of isthmic spondylolisthesis: pediatric and adult. Spine $J$ 2010; 10: 530-43.

8. Dubousset J. Treatment of spondylolysis and spondylolisthesis in children and adolescents. Clin Orthop Relat Res 1997; 77-85. 
9. Boissiere L, Perrin G, Rigal J, et al. Lumbar-sacral fusion by a combined approach using interbody PEEK cage and posterior pedicle-screw fixation: Clinical and radiological results from a prospective study. Orthop Traumatol Surg Res 2013; 99: 945-51.

10. Zhang C, Sun T, Tian R, et al. [Comparative study on two surgical treatment of isthmic spondylolisthesis]. Zhongguo Xiu Fu Chong Jian Wai Ke Za Zhi 2015; 29: 179-83.

11. Luo J, Cao K, Yu T, et al. Comparison of Posterior Lumbar Interbody Fusion Versus Posterolateral Fusion for the Treatment of Isthmic Spondylolisthesis. Clin spine Surg 2017; 30: E915-22.

12. Tye EY, Tanenbaum JE, Alonso AS, et al. Circumferential fusion: a comparative analysis between anterior lumbar interbody fusion with posterior pedicle screw fixation and transforaminal lumbar interbody fusion for L5-S1 isthmic spondylolisthesis. Spine J. Epub ahead of print August 15, 2017. DOI: 10.1016/j.spinee.2017.08.227.

13. Farah K, Graillon T, Rakotozanany P, et al. Circumferential minimally invasive approach for low-grade isthmic spondylolisthesis: A clinical and radiological study of 43 patients. Orthop Traumatol Surg Res 2018; 104: 5759.

14. Koné N, Freitas Olim E, Coloma $P$, et al. [A continuous series of 27 adult patients treated for L5-S1 isthmic spondylolisthesis by combined approach: Clinical and radiological outcomes at 1 year follow-up]. Neurochirurgie 2017; 63: 74-80.

15. Tamburrelli FC, Meluzio MC, Burrofato A, et al. Minimally invasive surgery procedure in isthmic spondylolisthesis. Eur Spine J 2018; 27: 237-43.

16. Noorian S, Sorensen K, Cho W. A systematic review of clinical outcomes in surgical treatment of adult isthmic spondylolisthesis. Spine J. Epub ahead of print May 7, 2018. DOI: $10.1016 /$ j.spinee.2018.04.022

17. Jacobs WCH, Vreeling A, De Kleuver M. Fusion for lowgrade adult isthmic spondylolisthesis: A systematic review of the literature. Eur Spine J 2006; 15: 391-402.

18. Carragee EJ. Single-level posterolateral arthrodesis, with or without posterior decompression, for the treatment of isthmic spondylolisthesis in adults. A prospective, randomized study. J Bone Joint Surg Am 1997;7 9: 117580 . 